\title{
cAMP Responsive Element-Binding Protein Phosphorylation Is Necessary for Perirhinal Long-Term Potentiation and Recognition Memory
}

\author{
E. Clea Warburton, ${ }^{1}$ Colin P. J. Glover, ${ }^{2}$ Peter V. Massey, ${ }^{1}$ Humin Wan, ${ }^{1}$ Ben Johnson, ${ }^{1}$ Alison Bienemann, ${ }^{2}$ \\ Ule Deuschle, ${ }^{4}$ James N. C. Kew, ${ }^{4}$ John P. Aggleton, ${ }^{3}$ Zafar I. Bashir, ${ }^{1}$ James Uney, ${ }^{2}$ and Malcolm W. Brown ${ }^{1}$ \\ ${ }^{1}$ Medical Research Council Centre for Synaptic Plasticity, Department of Anatomy, University of Bristol, Bristol BS8 1 TD, United Kingdom, ${ }^{2}$ The Henry \\ Wellcome Laboratories for Integrative Neuroscience and Endocrinology, Bristol BS1 3NY, United Kingdom, ${ }^{3}$ School of Psychology, University of Cardiff, \\ Cardiff CF10 3YG, United Kingdom, and 4 Preclinical CNS Research, F. Hoffmann-La Roche, CH-4070 Basel, Switzerland
}

\begin{abstract}
We established the importance of phosphorylation of cAMP responsive element-binding protein (CREB) to both the familiarity discrimination component of long-term recognition memory and plasticity within the perirhinal cortex of the temporal lobe. Adenoviral transduction of perirhinal cortex (and adjacent visual association cortex) with a dominant-negative inhibitor of CREB impaired the preferential exploration of novel over familiar objects at a long $(24 \mathrm{~h})$ but not a short $(15 \mathrm{~min})$ delay, disrupted the normal reduced activation of perirhinal neurons to familiar compared with novel pictures, and impaired long-term potentiation of synaptic transmission in perirhinal slices. The consistency of these effects across the behavioral, systems, and cellular levels of analysis provides strong evidence for involvement of CREB phosphorylation in synaptic plastic processes within perirhinal cortex necessary for long-term recognition memory.
\end{abstract}

Key words: temporal lobe; plasticity; familiarity discrimination; viral transduction; hippocampus; Fos

\section{Introduction}

The reported experiments were designed to determine the role of the phosphorylation of cAMP responsive element (CRE)binding protein (CREB) in perirhinal-dependent plasticity and recognition memory. The perirhinal cortex $(\mathrm{PRH})$ of the temporal lobe has major advantages for studying neural substrates of memory, especially for studies involving multiple levels of analysis. First, the region is essential to visual recognition memory, because tasks that depend on familiarity discrimination for their solution are severely impaired by ablations including perirhinal cortex in both rats and monkeys (Zola-Morgan et al., 1989; Ennaceur and Aggleton, 1997; Gaffan and Murray, 1992; Meunier et al., 1993, 1996; Suzuki et al., 1993; Mumby and Pinel, 1994; Murray, 1996; Buffalo et al., 2000; Winters et al., 2004). Second, the substrate for general long-term familiarity discrimination has been identified as the reduction in the responses of perirhinal neurons that occurs when a stimulus is re-encountered (Brown et al., 1987; Brown and Xiang, 1998; Brown and Aggleton, 2001; Brown and Bashir, 2002). In justification of this claim, it should be noted that other forms of repetition-related neuronal response changes observed in perirhinal cortex, such as response enhance-

\footnotetext{
Received Feb. 7, 2005; revised May 9, 2005; accepted May 17, 2005.

This work was supported by the Medical Research Council and the Biotechnology and Biological Sciences Research Council. We are grateful for the help and expertise of J. Robbins, B. Fry, and C. Narduzzo.

Correspondence should be addressed to Prof. M.W. Brown at the above address. E-mail:m.w.brown@bris.ac.uk.

J. N. C. Kew's present address: Psychiatry Centre of Excellence for Drug Discovery, GlaxoSmithKline, Harlow CM19 $5 A W$, UK.

DOI:10.1523/JNEUROSCI.0506-05.2005

Copyright $\odot 2005$ Society for Neuroscience $\quad 0270-6474 / 05 / 256296-08 \$ 15.00 / 0$
}

ments (Miller and Desimone, 1994) or slowly developing changes when stimuli are repeated tens of times (Holscher et al., 2004), do not provide a potential substrate for single-exposure familiarity discrimination of unrewarded stimuli; however, response reductions do (Brown and Xiang, 1998). Third, this change in responsiveness is sufficiently large that it can be demonstrated using Fos immunohistochemical imaging under closely controlled conditions (Zhu et al., 1995, 1996; Wan et al., 1999). Fourth, the site of the initial underlying plastic change is known to be the perirhinal cortex and/or the adjacent cortex of area TE, because the response reduction is first generated there (Brown and Xiang, 1998; Xiang and Brown, 1998). Fifth, theoretical network modeling has shown that synapse-specific plasticity involving mechanisms such as those underlying long-term potentiation (LTP) or longterm depression (LTD) could underlie familiarity discrimination (Bogacz et al., 2001; Bogacz and Brown, 2003). Hence, the mechanisms underlying LTP and LTD provide potential candidate mechanisms for processes effecting recognition memory. Sixth, such synaptic plasticity can be produced and thus studied in perirhinal brain slices (Ziakopoulos et al., 1999; Cho et al., 2000).

Investigation of the role of CREB phosphorylation was chosen for the following reasons. Previous work has associated CREBactivated transcriptional processes with learning and memory (Bourtchuladze et al., 1994; Liu and Graybiel, 1996; Silva et al., 1998; Dalley et al., 1999; Kogan et al., 2000; Genoux et al., 2002), but the role of such processes in perirhinal-dependent recognition memory has not been investigated. Additionally, previous work implicates CREB-activated pathways in synaptic plasticity (Deisseroth et al., 1996; Silva et al., 1998; Ahn et al., 1999), but 
their role in perirhinal plasticity is unknown. Moreover, CREB phosphorylation can lead to activation of the immediate early gene c-fos (Ahn et al., 1998; Silva et al., 1998), expression of whose protein products (Fos) provides an accurate marker for recognition memory processes (Zhu et al., 1995, 1996; Wan et al., 1999, 2004; Warburton et al., 2003). However, the role of CREB phosphorylation in such Fos changes in the perirhinal cortex has not been studied.

To effect gene transcription, CREB must be phosphorylated and dimerized. Transcription may therefore be blocked by causing CREB to bind to a modified protein that will prevent the resultant heterodimer from binding to DNA. Such a block can be achieved by using a dominant-negative inhibitor of CREB plus its close relatives (denoted A-CREB); this produces hyperstable heterodimers that will not bind to DNA (Ahn et al., 1998). This A-CREB construct consists of an acidic amphipathic extension that replaces the natural basic region fused on to the $\mathrm{N}$ terminus of the CREB leucine domain. The acidic extension interacts with the basic region of endogenous CREB, forming a coiled-coil extension of the leucine zipper and preventing CREB from binding DNA and initiating CRE-mediated transcription. The CREB/ACREB heterodimer was demonstrated to be 3300 -fold more stable than CREB homodimers. We investigated previously the specificity of A-CREB function using HeLa and primary hippocampal neurons. These data showed that the activation of adenylate cyclase by forskolin was totally inhibited by the viralmediated expression of A-CREB, strongly suggesting that the CREB-2 repressor was not itself inactivated (Glover et al., 2004). Furthermore, Ahn et al. (1998) showed the following: (1) A-CREB interacts selectively with CREB and not other basic leucine zipper (B-ZIP) domains, and (2) A-CREB inhibition of cAMP-sensitive gene expression was via the inhibition of the DNA-binding activity of CREB and not via the inhibition of other B-ZIP dimerization partners. Together, these results establish that A-CREB will bind to close family members such as ATF-1 (activating transcription factor-1) and CREM (cAMP responsive element modulator) but will not bind to other B-ZIP proteins. A-CREB may be expressed in cells in a specific region through localized injection of an adenoviral (Ad) vector in the adult. Exploiting this, we test the hypotheses that adenoviral transduction of perirhinal cortex with A-CREB disrupts both long-term recognition memory and perirhinal plasticity (LTP). Additionally, by using such adenoviral transduction, we test the hypotheses that differential perirhinal neuronal activity (as measured by Fos immunohistochemistry) in response to novel and familiar stimuli underlies familiarity discrimination and that these differential responses rely on a synaptic plastic mechanism used in LTP.

\section{Materials and Methods}

\section{Subjects}

Male pigmented rats (total, $n=64$; DA strain; Bantin and Kingman, Hull, UK) weighing 200-230 g at the start of the experiment were used in all experiments. Each animal was used in only one of experiments A, B, or $\mathrm{C}$ [although in $\mathrm{B}$, measurements of Fos and phosphorylated CREB (pCREB) were made on the same animals]. The animals were housed under a $12 \mathrm{~h}$ light/dark cycle (light phase, 6:00 P.M. to 6:00 A.M.). Behavioral training and testing were conducted during the dark phase of the cycle. All animal procedures were approved by the local Ethical Committee and performed in accordance with United Kingdom Animals Scientific Procedures Act (1986) and associated guidelines. All efforts were made to minimize the suffering and the number of animals used.

All statistical tests were two tailed unless otherwise stated and used a significance level of 0.05 .

\section{Adenoviral preparation}

Recombinant E1-deleted Ad constructs were produced according to standard techniques (Harding et al., 1998). The cDNA for the reporter construct, enhanced green fluorescent protein (EGFP), was excised from pEGFP1 (Clontech, Cambridge, UK) using HindIII and XbaI and inserted into the corresponding sites in the multiple cloning site of the plasmid pXCXCMV (Harding et al., 1998). The cDNAs for CREB and the dominant-negative A-CREB were expressed bicistronically with EGFP by cloning each individually into the same Ad transfer plasmid. Recombinant virus was generated by homologous recombination in human embryonic kidney 293 cells (Microbix Biosystems, Toronto, Canada), grown to high titer, and purified by $\mathrm{CsCl}$ density gradient centrifugation. Viral titer was determined by plaque assay: Ad-CMV-EGFP was $1 \times 10^{10}$ $\mathrm{pfu} / \mathrm{ml}$, and Ad-CMV-A-CREB was $1 \times 10^{10} \mathrm{pfu} / \mathrm{ml}$.

\section{Transduction}

Before surgery, all animals were deeply anesthetized by intraperitoneal injection $(60 \mathrm{mg} / \mathrm{kg})$ of pentobarbitone sodium (Sagatal; Rhône Mérieux, France) and then placed in a stereotaxic head-holder (David Kopf Instruments, Tujunga, CA) with the nose bar at $+5.0 \mathrm{~mm}$ above the ear bars. The scalp was cut and retracted to expose the skull. Craniotomies were made directly above the target region, and the dura was cut to expose the cortex.

Animals received $2.0 \mu \mathrm{l}$ of viral suspension [Ad-CMV-EGFP control or the dominant-negative Ad-CMV-A-CREB (i.e., A-CREB)] via a handpulled glass needle using a Harvard infusion pump, using a flow rate of $0.2 \mu \mathrm{l} / \mathrm{min}$ over $10 \mathrm{~min}$. One injection on each side was directed into the perirhinal cortex using the following stereotaxic coordinates relative to bregma: anteroposterior, -4.8 ; lateral, \pm 6.8 ; height -9.1 . Preliminary experiments indicated that a single injection was adequate to transduce the posterior part of the perirhinal cortex within which the significant changes in Fos expression related to stimulus familiarity are localized (Zhu et al., 1996; Wan et al., 1999) and that tissue damage was minimized by using a single, rather than multiple, injection. At the completion of surgery, the skin was sutured, and an antibiotic powder (Acramide; Dales Pharmaceuticals, Skipton, UK) was applied. All animals then received 5 $\mathrm{ml}$ of glucose saline (subcutaneously) and systemic analgesia. After surgery, animals were allowed 2 weeks to recover before the start of handling and, as necessary, behavioral habituation. Experiments were therefore conducted 3-4 weeks after injection of adenovirus. Adenoviral vectors have been shown to continue to mediate gene expression in vivo for $>3$ months (Geddes et al., 1997); at 3 months after the AdCMV-A-CREBEGFP bicistronic vector was injected into the brain, EGFP expression could still be detected using fluorescence microscopy.

\section{Experiment A: object recognition}

Apparatus. Exploration occurred in an open-topped arena $(90 \times 100 \mathrm{~cm})$ with $50 \mathrm{~cm}$ wood walls and a scaffold covered with black cloth to a height of $150 \mathrm{~cm}$, so that no external stimuli could be seen during the experiment. The floor was covered with sawdust. An overhead camera and a video recorder were used to monitor and record the animal's behavior for subsequent analysis. The stimuli presented were triplicate copies of objects made of glass or plastic that varied in shape, color, and size $(9 \times$ $8 \times 5 \mathrm{~cm}$ to $25 \times 10 \times 5 \mathrm{~cm}$ ) and were too heavy for the animal to displace.

Training. After being handled for 1 week, each of the 36 animals (18 A-CREB and 18 EGFP control) was habituated to the arena without stimuli for 10-15 min daily for $2 \mathrm{~d}$ before the commencement of the spontaneous recognition procedure. This procedure was composed of an acquisition or sample phase followed by two test phases at delays of 15 min and $24 \mathrm{~h}$. In the sample phase, duplicate copies of an object (e.g., A1 and A2) were placed near the two corners at either end of one side of the arena $(10 \mathrm{~cm}$ from each adjacent wall). The animal was placed into the arena facing the center of the opposite wall and then allowed a total of either $40 \mathrm{~s}$ of exploration of A1 and A2 or $4 \mathrm{~min}$ in the arena. Exploratory behavior was defined as the animal directing its nose toward the object at a distance of $<2 \mathrm{~cm}$. Any other behavior, such as looking around while sitting on or resting against the object, was not considered to be exploration. Exploration was scored by an observer blind to the rat's treat- 
ment. In the first test phase after a delay of $15 \mathrm{~min}$, the animal was replaced in the arena for $3 \mathrm{~min}$ and presented with two objects in the same positions: one object (A3) was the third copy of the triplicate set of the objects used in the sample phase, and the other was a novel object (B3). In the second test phase after a delay of $24 \mathrm{~h}$, the choice was between A3 and C3. Both objects had been washed with ethanol after the first test, and all objects were washed before procedures began with another rat. The positions of the objects in the test phases and the objects used as novel or familiar were counterbalanced between the animals in a group and between the control and A-CREB-treated groups. Exploration was scored by an observer blind to the rat's treatment. During the first $2 \mathrm{~min}$ of the test phase, the difference in time exploring the novel compared with the familiar object divided by the time spent exploring both objects was calculated as the discrimination ratio (Dix and Aggleton, 1999).

Histology. At the end of the behavioral experiments, each rat was deeply anesthetized with Euthatal (Rhône-Mérieux, Hertfordshire, UK) and perfused with $0.1 \mathrm{M}$ phosphate buffer $(\mathrm{PB})$ and $50 \mathrm{~mm}$ sodium fluoride $(\mathrm{NaF})$ followed by $\mathrm{PB}-\mathrm{NaF}$ containing $4 \%$ paraformaldehyde, $\mathrm{pH}$ 7.4. The brains were postfixed in paraformaldehyde for a minimum of $2 \mathrm{~h}$ before being transferred to $30 \%$ sucrose in $0.1 \mathrm{M} \mathrm{PB}$ and left overnight. Coronal sections were cut at $40 \mu \mathrm{m}$ on a cryostat. Alternate sections were Nissl stained with cresyl violet or mounted directly onto slides using Vectorshield (Vector Laboratories, Burlingame, CA), coverslipped, and examined using a Leica (Nussloch, Germany) DMR upright fluorescent microscope.

\section{Experiment B: immunohistochemical staining for Fos and pCREB}

Subjects, apparatus, and stimuli. Novel and familiar pictures were presented to each rat simultaneously under closely controlled conditions (Zhu et al., 1996; Wan et al., 1999) so that the evoked neuronal activation as measured by Fos or pCREB could be compared. Bilateral viral transduction was with A-CREB in eight rats and with EGFP in eight controls. Each rat was trained in a paired viewing chamber $(30 \times 30 \times 35 \mathrm{~cm})$. The top of the chamber was open, the bottom and the sides were black, and the front was transparent (Perspex) with a central observing hole, $3 \mathrm{~cm}$ in diameter, $6 \mathrm{~cm}$ above the floor. In the chamber, $4.5 \mathrm{~cm}$ to either side of the observing hole ran two small barriers ( $12 \mathrm{~cm}$ long $\times 9 \mathrm{~cm}$ high); these kept the rat's body at $90^{\circ}$ to the front screen when its head was in the observing hole. When the rat's head was positioned in the observing hole, an infrared beam was interrupted, thus signaling the computer [Viglen (Alperton, Middlesex, UK) P5-100] to start a trial. After a variable interval of 3-4 s, provided the head remained in the hole, two pictures (each $15 \times 12 \mathrm{~cm}$ ) were shown simultaneously for $4.5 \mathrm{~s}$, one on each of two computer monitors (model 4Vlr; AOC Spectrum, Los Angeles, CA) placed $30 \mathrm{~cm}$ from the observing hole. A black partition ensured that the rat's left eye could not see the right monitor screen and his right eye could not see the left screen. After the pictures had been seen for $4 \mathrm{~s}$, a drop of blackcurrant juice was delivered by a metal tube that the rat could just reach and lick. This paired viewing procedure (Zhu et al., 1996) ensured that the rat's behavior (which was monitored by camera and was video recorded) was the same for the novel and familiar pictures. The visual pictures were two-dimensional representations of single objects taken from Microsoft (Seattle, WA) Clip-Art and were varied in color and shape.

Training. During training, the rats were allowed ad libitum access to water for $2 \mathrm{~h}$ each day. Each rat was pretrained for $3 \mathrm{~d}$ without stimulus presentation to go to the observing hole for juice reward. The subsequent training period lasted $6 \mathrm{~d}$ with one morning and one afternoon training session per day. In the morning session, one set of 30 pictures, the "repeat set" (RPT set), was presented to each eye. On each trial, the left and right eyes saw simultaneously a different picture from the set, so that, over 30 trials, each eye saw all of the pictures once. In the afternoon training session, $3 \mathrm{~h}$ after the end of the morning session, one eye was exposed to the RPT set of pictures while the other eye was exposed to a set of 30 novel pictures; again, picture presentations were made simultaneously to each eye. A different set of 30 novel pictures was shown each afternoon to familiarize the animal with seeing novel and familiar pictures simultaneously, but the same RPT set of stimuli was used each day. Again, the side of presentation of the novel and RPT sets was counterbalanced across days. Thus, the different sets of novel and the RPT set of stimuli were presented such that by the end of the experiment, each eye had seen the same number of novel and repeated stimuli. The final set of novel pictures (the NOV set) was shown with the RPT set on the afternoon of day 6, with one eye seeing each set. The RPT set for half of the animals was the NOV set for the other half of the animals, so that the particular pictures used were counterbalanced across animals. Additionally, whether the left eye or the right eye was exposed to the NOV set was counterbalanced across animals. For there to be evidence of memory, information about the previous occurrence of the RPT set had to have been retained for a minimum of $3 \mathrm{~h}$ (the time between the last morning and the final test).

Immunohistochemical procedures. Each rat was deeply anesthetized with pentobarbitone and perfused with $0.1 \mathrm{M}$ phosphate buffer and 50 mM sodium fluoride followed by $\mathrm{PB}-\mathrm{NaF}$ containing $4 \%$ paraformaldehyde, $\mathrm{pH}$ 7.4, $1.5 \mathrm{~h}$ after the final set of stimuli had been shown. After perfusion, the brain was removed and placed in $4 \%$ paraformaldehyde in $\mathrm{PB}-\mathrm{NaF} 4$ for $12 \mathrm{~h}$, followed by $24 \mathrm{~h}$ in $30 \%$ sucrose in PB-NaF. Coronal sections $(30 \mu \mathrm{m})$ were cut on a cryostat, and floating sections were processed for immunocytochemistry with the avidin-biotin complex (ABC; Vector Laboratories) method. The secondary antibody was biotinylated goat anti-rabbit (Vector Laboratories). 3,3'-Diaminobenzine was used for visualization. After cutting the brain, sections were washed and preincubated in blocking serum $\left[0.3 \% \mathrm{H}_{2} \mathrm{O}_{2}\right.$ in PBST (PB-NaF plus $0.9 \%$ $\mathrm{NaCl}$ plus $0.2 \%$ Triton X-100)], followed by incubation in PBST plus $3 \%$ BSA. Sections were then incubated with either a polyclonal rabbit primary antibody for Fos (Ab-5; 1:5000; Calbiochem, La Jolla, CA) in PBST for $48 \mathrm{~h}$ or pCREB (1:1000; Upstate Biotechnology, Lake Placid, NY) for $24 \mathrm{~h}$ in $0.1 \mathrm{M}$ Tris- $\mathrm{HCl}$ containing $0.9 \% \mathrm{NaCl}$ and Triton $\mathrm{X}-100$ and $\mathrm{NaF}$.

Data analysis. The automated counting of stained nuclei was performed using an image analysis system (Seescan, Cambridge, UK) (McCabe and Horn, 1994; Zhu et al., 1995). Sections were visualized using a Zeiss (Thornwood, NY) Axioskop light microscope, and images $(512 \times 512$ pixels $)$ were displayed on the computer screen at an effective magnification of $200 \times$ and captured at a 256 gray level resolution. All processing and counting was done blind. Adjacent sections were stained for Fos or pCREB. Sections were only accepted for analysis if staining was uniform on both sides and artifact-free for both Fos and pCREB. Accordingly, data were used from five rats in the A-CREB group and from four rats in the EGFP control group. pCREB staining failed in one batch containing two A-CREB and two EGFP rats; nonuniform pCREB staining led to the rejection of one additional A-CREB and two EGFP controls. As in previous work (Zhu et al., 1995, 1996; Wan et al., 1999; Warburton et al., 2003), a nucleus was counted if one or more pixels was $>30$ gray levels darker than the darkest pixel surrounding the image of the nucleus. Counts above threshold were obtained from the right and left hemispheres for rectangular areas $(0.94 \times 0.67 \mathrm{~mm})$ from two sections for each brain region. This rectangular area included all cortical layers. Stereological corrections were not used, because relative changes were all that were sought. For statistical analysis, each count was normalized by dividing by the mean of the total counts for the area across both hemispheres for each rat. The normalized counts were then square root transformed and subjected to a repeated-measures ANOVA with factors rat, area, transduction treatment, and stimulus repetition (NOV/RPT).

\section{Experiment C: measurement of LTP in perirhinal slices}

Material. Data were obtained from slices of perirhinal cortex prepared from 12 bilaterally infused rats, six A-CREB and six viral controls. Animals were anesthetized with a halothane/oxygen mixture and decapitated, and the brain was rapidly removed and placed in ice-cold artificial CSF (aCSF; bubbled with $95 \% \mathrm{O}_{2} / 5 \% \mathrm{CO}_{2}$ ) composed of the following (in mM): $124 \mathrm{NaCl}, 3 \mathrm{KCl}, 26 \mathrm{NaHCO}_{3}, 1.25 \mathrm{NaH}_{2} \mathrm{PO}_{4}, 2 \mathrm{CaCl}_{2}, 1$ $\mathrm{MgSO}_{4}$, and $10 \mathrm{D}$-glucose. A midsagittal section was made, and the rostral and caudal parts of the brain were removed by single scalpel cuts made at $\sim 45^{\circ}$ to the dorsoventral axis and each hemisphere glued by its caudal end to a vibroslice stage (Campden Instruments, Sileby, UK). Slices $(400 \mu \mathrm{m})$, which included perirhinal, entorhinal, and temporal cortices were stored submerged in aCSF $\left(20-25^{\circ} \mathrm{C}\right)$ for $1-2 \mathrm{~h}$ before 

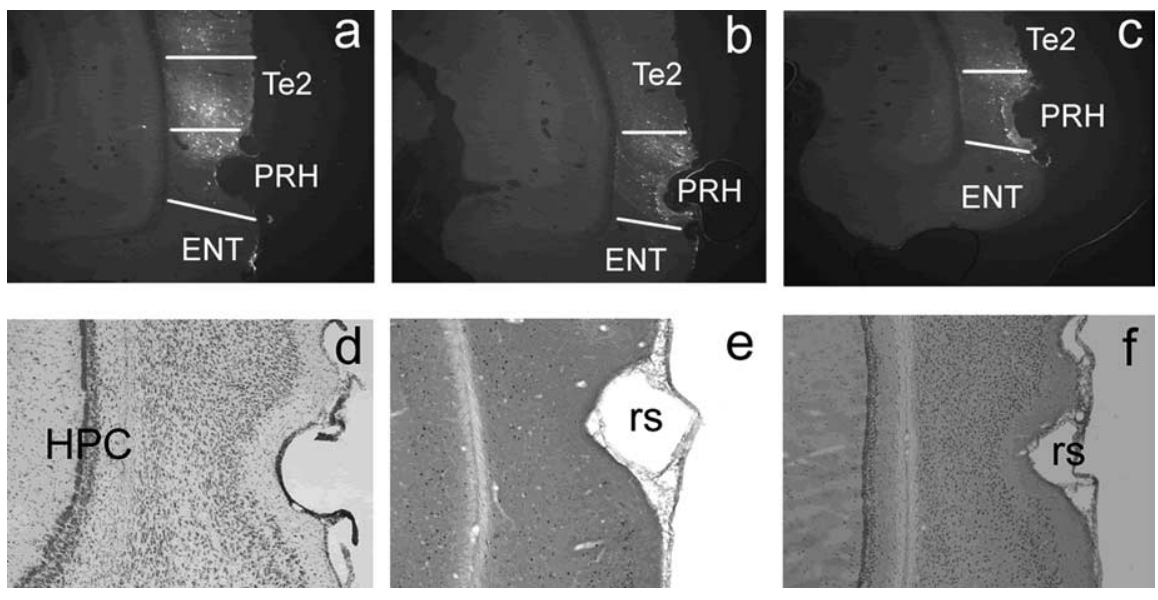

Figure 1. Perirhinal histology. $\boldsymbol{a}-\boldsymbol{c}$, Extent of EGFP expression in the perirhinal cortex and area Te2 after infusion of adenoviral vectors. The white lines indicate the boundaries of the PRH, area Te2, and entorhinal cortex (ENT). Distance behind bregma: $\boldsymbol{a}$ $-4.8 \mathrm{~mm} ; \boldsymbol{b},-5.6 \mathrm{~mm} ; \boldsymbol{c},-6.7 \mathrm{~mm}$. $\boldsymbol{d}$, Nissl-stained section of the perirhinal cortex and area Te2 showing no damage to the tissue caused by the infusion. The positions of the hippocampus (HPC) and rhinal sulcus (rs) are indicated. $\boldsymbol{e}$, Example section showing Fos-stained nuclei. $f$, Example section showing pCREB-stained nuclei.

transferring to the recording chamber. A single slice was placed in a submerged recording chamber $\left(28-30^{\circ} \mathrm{C}\right.$; flow rate, $\left.\sim 2 \mathrm{ml} / \mathrm{min}\right)$ when required.

Recording. Standard extracellular recording techniques were used to monitor evoked field responses from layers II/III (Ziakopoulos et al., 1999). One stimulating electrode was placed dorsorostrally on the temporal cortex side (areas 35/36) and one ventrocaudally on the entorhinal cortex side (area 35/entorhinal cortex) of the rhinal sulcus. Stimuli (constant voltage) were delivered alternately to the two stimulating electrodes (each electrode, $0.033 \mathrm{~Hz}$ ). The amplitude of the evoked field EPSPs was measured and expressed relative to the preconditioning baseline. Baseline responses were set to $\sim 70 \%$ of the maximal response. Highfrequency stimulation (HFS: four $1 \mathrm{~s}$ trains of $100 \mathrm{~Hz}$ stimulation with a $10 \mathrm{~s}$ interval between each train) was delivered to elicit LTP.

Data analysis. Data were only analyzed from one slice per rat (i.e., six A-CREB and six EGFP controls). Data were recorded using an Axoclamp 2B amplifier (Molecular Devices, Union City, CA), monitored, and analyzed on-line and reanalyzed off-line (Anderson and Collingridge, 2001). Because there were no significant differences between the results of stimulating the temporal and entorhinal sides, the data were pooled. The effects of conditioning stimulation were followed for $>45 \mathrm{~min}$ after induction of LTP.

\section{Results}

\section{Histology}

Both control and A-CREB adenoviral vectors were tagged with EGFP. Infusion of the vectors produced localized transgene expression within area $\mathrm{Te} 2$ and $\mathrm{PRH}$, particularly dorsal PRH. There was some limited spreading back along the needle track into the adjacent auditory cortex. A few scattered EGFP-positive cells were seen in the ventral hippocampus on one side or the other in approximately one-third of the animals; these animals showed no behavioral differences from those in which no such staining was observed. In no animal was there staining of hippocampal cells in both hemispheres. Figure $1 a-c$ shows the EGFP expression after the completion of the behavioral experiments in representative brain sections. Infusion of the adenoviral vectors produced minimal tissue damage, as assessed by cresyl violet staining (Fig. 1d).

Histological examination of the infusion site showed that all of the rats infused with Ad-CREB or Ad-EGFP and tested in the object recognition task or the paired viewing procedure had bilaterally accurate infusion sites within the perirhinal cortex and area Te2. The slices used in the LTP experiments were taken from this same region.

\section{Experiment A: recognition memory} measured by preferential exploration of novel objects

Familiarity discrimination was impaired at a long $(24 \mathrm{~h})$ but not short $(15 \mathrm{~min})$ delay by microinjection bilaterally into the perirhinal cortex of adenovirus causing expression of A-CREB (Fig. 2). For control rats that received bilateral perirhinal microinjections of adenovirus expressing only EGFP, the normal, greater exploration of a novel than a previously sampled (i.e., familiar) object was seen at both the long and the short delays.

In detail, ANOVA revealed a significant adenoviral treatment by time delay interaction $\left(F_{(1.34)}=4.09 ; p=0.05\right)$. Additional analysis demonstrated a significant difference between the A-CREB and control rats during the recognition memory test that occurred at a delay of $24 \mathrm{~h}$ after the sampling period $\left(F_{(1,34)}=5.58 ; p<0.025\right)$ but not when the delay was only $15 \mathrm{~min}$ $\left(F_{(1,34)}<1.0 ; p>0.1\right)$. At the 15 min delay, both the control and the A-CREB rats spent significantly more time exploring a novel than a familiar object $\left(t_{17}=4.77\right.$ and 4.22 , respectively; each, $p<$ $0.001)$. In contrast, at the $24 \mathrm{~h}$ delay, whereas the control rats continued reliably to discriminate the novel from the familiar object $\left(t_{17}=4.31 ; p<0.001\right)$, the A-CREB rats failed to show such discrimination $\left(t_{17}=0.59 ; p>0.1\right)$.

There was no evidence of an effect of A-CREB treatment on general explorative behavior. The total time required by the rats to complete $40 \mathrm{~s}$ of exploration of the two copies of the object presented for familiarization during the sampling period was not significantly $\left(F_{(1,34)}<1.0 ; p>0.1\right)$ different for the control $($ mean $=170 \pm 14 \mathrm{~s})$ and A-CREB animals $($ mean $=185 \pm 14 \mathrm{~s})$.

\section{Experiment B(1): differential neuronal activation by novel} and familiar pictures measured by Fos

The normal pattern of greater Fos expression produced by viewing novel rather than familiar pictures was disrupted by microinjection bilaterally into the perirhinal cortex of adenovirus causing expression of A-CREB (Fig. 3).

When one eye is exposed to novel stimuli at the same time the other is exposed to repeatedly presented (familiar) stimuli using a paired viewing procedure, previous studies (Zhu et al., 1996; Wan et al., 1999) have established reliable differences between the two hemispheres in counts of Fos-stained nuclei in the PRH and neighboring visual association cortex (area Te2): counts are higher in the hemisphere opposite the novel stimuli than that opposite the familiar stimuli. The final test comparison of the Fos activation produced by the novel and familiar stimuli is $3 \mathrm{~h}$ after the previous viewing of the familiar stimuli (i.e., the familiar stimuli must be remembered for $\geq 3 \mathrm{~h}$ ).

Using this procedure, Fos counts were made in $\mathrm{PRH}$ and $\mathrm{Te} 2$ in rats with microinjections made bilaterally into the perirhinal cortex of control EGFP and A-CREB expressing adenovirus (Fig. $1 e$ ). As in previous work, the Fos counts were normalized across hemispheres and rats. Each data value was then transformed by taking its square root to improve the symmetry of the distribution. These transformed counts were compared using repeated- 


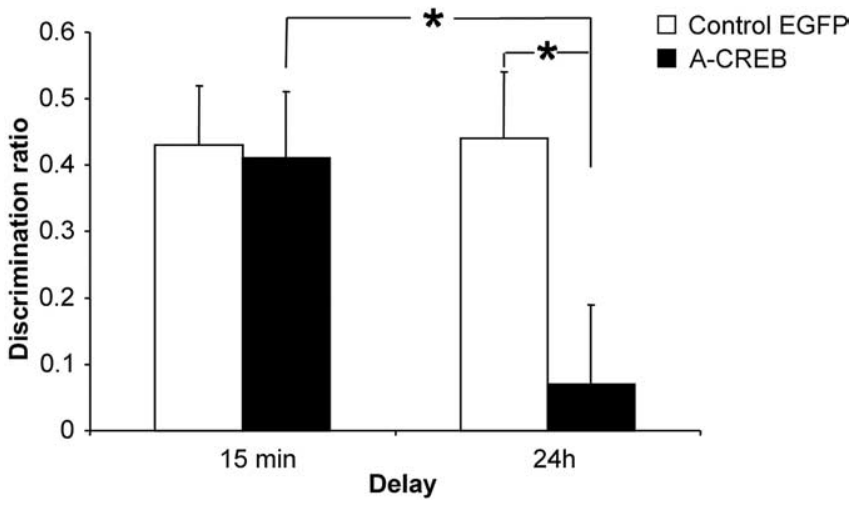

Figure 2. Impairment of familiarity discrimination at a long (24 h) but not a short (15 min) delay in A-CREB but not viral control animals. The histogram bars indicate the mean relative time ( \pm SEM) spent exploring the novel compared with the previously seen object (discrimination ratio) after transduction with adenovirus-expressing A-CREB or adenovirus-expressing EGFP (control). Note the lack of discrimination of the A-CREB group at the $24 \mathrm{~h}$ delay $\left({ }^{*} p<\right.$ 0.05).

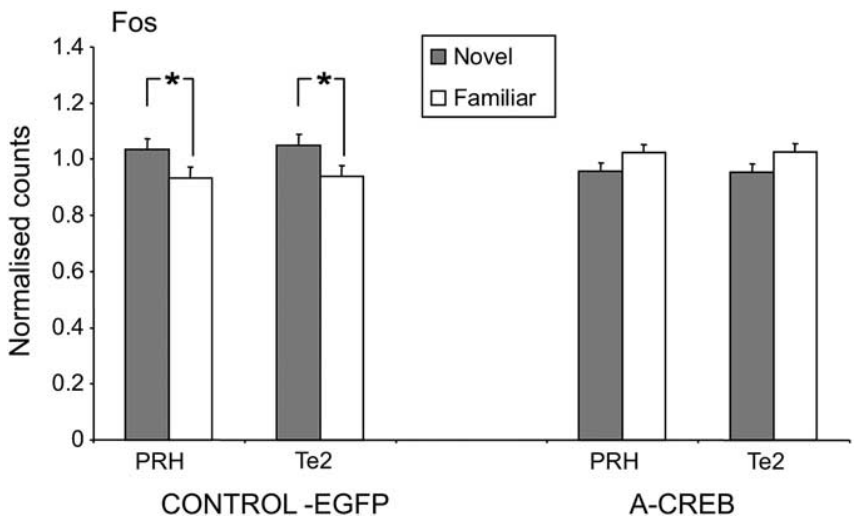

Figure 3. Disruption of the normal pattern of Fos counts in the A-CREB-transduced (A-CREB) but not in the EGFP-transduced control (CONTROL-EGFP) animals. Note that, in the control but not the A-CREB animals, the Fos counts were significantly ( ${ }^{*} p<0.05$ ) higher in PRH and Te2 for novel compared with familiar stimuli.

measures ANOVA with factors treatment, stimulus repetition, area, and rat. There was a highly significant interaction between adenoviral treatment and stimulus repetition $\left(F_{(1,144)}=13.17\right.$; $p<0.001)$. No other interactions were significant. In control rats, as expected from previous studies, mean counts for novel were significantly higher than for familiar stimuli $(p<0.005$; onetail), with the difference being significant for both $\mathrm{PRH}$ and Te2 (each, $p<0.05$; one-tail) (Fig. 3 ). In contrast, in the A-CREB rats, mean counts for novel were significantly lower than for familiar stimuli $(p<0.05)$, although the difference did not reach significance for either PRH or Te2 taken separately (Fig. 3).

\section{Overall counts}

There was no evidence that the different adenoviral treatments produced any effect on the neuronal activation evoked by visual stimuli when no account was taken of whether these stimuli had been shown previously. Thus, although the absolute, nonnormalized counts produced a highly significant interaction between treatment and stimulus repetition $\left(F_{(1,144)}=11.91 ; p<\right.$ 0.001 ), as found for the normalized counts, the mean Fos counts averaged for all stimuli, whether novel or previously presented, did not differ significantly $\left(F_{(1,144)}<1 ; p>0.1\right)$ between the
Table 1. Mean absolute Fos and pCREB counts ${ }^{a}$

\begin{tabular}{|c|c|c|c|c|}
\hline \multirow[b]{2}{*}{ Areas } & \multicolumn{2}{|c|}{ Control-EGFP } & \multicolumn{2}{|c|}{ A-CREB } \\
\hline & Novel & Familiar & Novel & Familiar \\
\hline \multicolumn{5}{|l|}{ Fos } \\
\hline PRH & 96 & 85 & 82 & 98 \\
\hline Te2 & 149 & 124 & 115 & 141 \\
\hline \multicolumn{5}{|l|}{$\mathrm{pCREB}$} \\
\hline PRH & 658 & 483 & 261 & 388 \\
\hline Te2 & 694 & 516 & 260 & 496 \\
\hline
\end{tabular}

${ }^{a}$ Counts above threshold in a $0.94 \times 0.67 \mathrm{~mm}$ frame (see Materials and Methods).

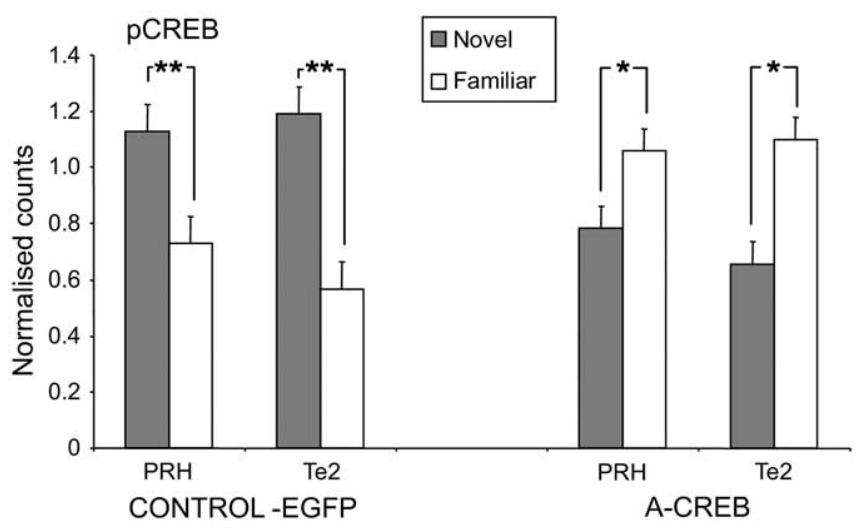

Figure 4. Disruption of the normal pattern of pCREB counts in the A-CREB-transduced (ACREB) but not in the EGFP-transduced control (CONTROL-EGFP) animals. Note that, in the control, the pCREB counts were significantly $\left({ }^{* *} p<0.01\right)$ higher in PRH and Te2 for novel compared with familiar stimuli. In the A-CREB-transduced animals, this pattern of activation was reversed $\left({ }^{*} p<0.05\right)$.

A-CREB and control groups, either overall or for any individual area (Table 1).

\section{Experiment B(2): differential neuronal activation by novel} and familiar pictures measured by $\mathrm{pCREB}$

The control pattern of greater pCREB expression produced by viewing novel rather than familiar pictures was disrupted by microinjection bilaterally into the perirhinal cortex of adenovirus causing expression of A-CREB (Fig. 4).

Counts of pCREB-stained nuclei were made for adjacent sections of PRH and Te2 to those assessed for Fos in the rats with microinjections made bilaterally into the perirhinal cortex of control EGFP and A-CREB expressing adenovirus (Fig. 1f). Thus, the effective memory delay was also $\geq 3 \mathrm{~h}$. As for Fos, counts were normalized across hemispheres and rats and then square root transformed before being compared with repeatedmeasures ANOVA. There was a highly significant interaction between adenoviral treatment and stimulus repetition $\left(F_{(1,144)}=\right.$ $49.16 ; p<0.001)$. No other interactions were significant. In control rats, mean counts for novel were significantly higher than for familiar stimuli ( $p<0.001)$, with the difference being significant for both PRH and Te2 (each, $p<0.005$ ) (Fig. 4). In contrast, in the A-CREB rats, mean counts for novel were significantly lower than for familiar stimuli $(p<0.001)$, and the difference was significant in both PRH and Te2 taken separately $(p<0.05)$ (Fig. 4).

\section{Overall counts}

In the A-CREB group overall, the mean pCREB absolute, nonnormalized count was highly significantly below that for the controls $\left(351 \pm 30\right.$ compared with $588 \pm 35 ; F_{(1,144)}=27.00 ; p<$ $0.001)$. The reductions were similar and significant in both the 


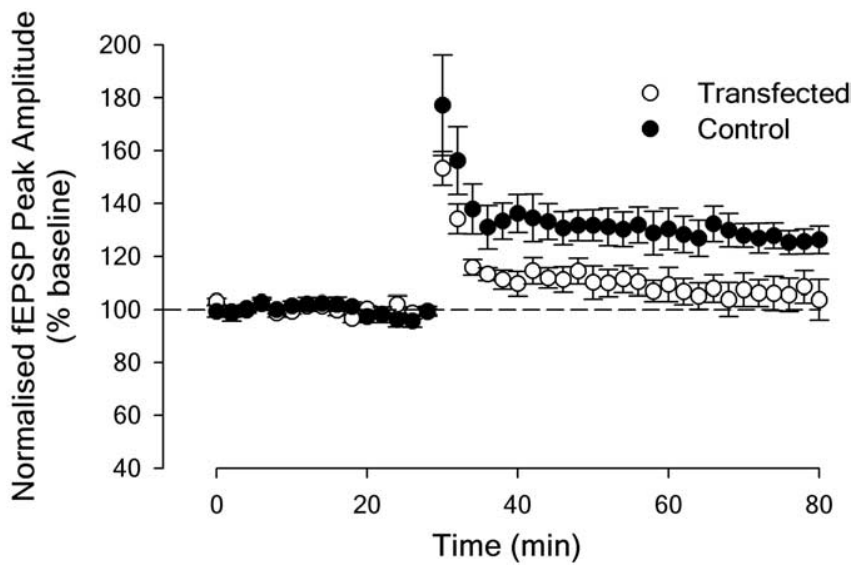

Figure 5. Impairment of LTP in slices of the perirhinal cortex taken from A-CREB-transduced animals. For these, high-frequency stimulation resulted in the induction of potentiation that declined to baseline values over the course of the experiment. In contrast, stable LTP was induced in the perirhinal cortex from control (EGFP-transduced) animals.

PRH and Te2 (respectively, to 57 and 62\% of control; $p<0.001$, for each) (Table 1). In addition, these absolute, non-normalized counts demonstrated a highly significant $\left(F_{(1,144)}=15.49 ; p<\right.$ $0.001)$ interaction between treatment and stimulus repetition, mirroring that for the normalized counts, as is to be expected given the reversal of the direction of effects between treatments (Table 1).

\section{Experiment C: perirhinal LTP}

LTP was impaired in slices of the perirhinal cortex taken from rats microinjected with adenovirus causing the expression of A-CREB (Fig. 5).

The effects of adenoviral infection were sought on LTP induced in perirhinal slices maintained in vitro. After a stable baseline had been established for each of two inputs that were alternately stimulated, LTP was induced by HFS (four $1 \mathrm{~s}$ trains, 100 $\mathrm{Hz}$ ) (Ziakopoulos et al., 1999) applied to one input while baseline stimulation continued for the second input. Stable LTP was induced in control slices measured at both $15 \pm 5$ min (mean = $\left.33 \pm 7 \%, n=6 ; t_{5}=5.02, p<0.005\right)$ and $45 \pm 5 \min (26 \pm 5 \%$, $\left.n=6 ; t_{5}=5.07, p<0.005\right)$ after HFS. In the A-CREB slices, however, although there was significant LTP at $15 \pm 5 \mathrm{~min}(12 \pm$ $\left.5 \%, n=6 ; t_{5}=2.63, p<0.05\right)$, by $45 \pm 5 \mathrm{~min}$ after induction, potentials had returned to baseline $\left(6 \pm 6 \%, n=6 ; \mathrm{t}_{5}=1.07, p>\right.$ 0.1 ) (Fig. 5). The difference in the magnitude of the LTP between control and A-CREB groups for both the 15 and 45 min comparisons was significant $\left(t_{10}=2.59\right.$ and 2.62, respectively; $\left.p<0.05\right)$.

\section{Discussion}

The results establish that adenoviral transduction of the perirhinal cortex and adjacent visual association cortex (area Te2) with a dominant-negative construct (A-CREB) that prevents the interactions of pCREB with the nucleus disrupts long-term recognition memory as well as perirhinal plasticity. Additionally, the results provide evidence in support of the hypotheses that differential perirhinal neuronal activity (as measured by Fos immunohistochemistry) in response to novel and familiar stimuli underlies familiarity discrimination and that these differential responses rely on a synaptic plastic mechanism used in LTP.

The use of adenoviral transduction avoids problems concerning the interpretation of results arising from the use of nonregionally specific, noninducible, transgenic animals. Adenoviral transduction allows interference with the function of CREB to be confined to a localized region of the brain in the adult animal. Moreover, the lack of biochemical specificity and large-scale destruction of neurons and glia produced by conventional lesions is avoided (Neve, 1993). The functionality of the adenoviral A-CREB-expressing vector was established in a previous study: a CREB-expressing vector but not the A-CREB vector protected cells from excitotoxic and ischemic stress (Glover et al., 2004). Although adenoviral transduction has been used previously in behavioral studies, such as those investigating restoration of motor function in animal models of dopamine depletion (Umegaki et al., 1997; McMenamin et al., 2004), to our knowledge, this is the first time it has been used to demonstrate an interference with recognition memory.

Similarly, this is the first report that CREB phosphorylation within the perirhinal cortex is important for recognition memory, although CREB phosphorylation has been shown previously to be important to other mammalian memory processes (Bourtchuladze et al., 1994; Guzowski and McGaugh, 1997; Lamprecht et al., 1997; Kida et al., 2002; Pittenger et al., 2002; Bozon et al., 2003). The current findings also add to the evidence that recognition memory impairment does not require direct interference with the hippocampus. In addition, this is the first full report demonstrating the importance of CREB phosphorylation for perirhinal plasticity: LTP, particularly its maintenance, was impaired, as in other brain regions such as the hippocampus after interference with CREB phosphorylation (Pittenger et al., 2002; Balschun et al., 2003). Moreover, the experiments demonstrate not only that the viral transduction of the perirhinal cortex impairs recognition memory and perirhinal synaptic plasticity, they also establish that the differential neuronal activation generated in the perirhinal cortex by novel and familiar stimuli is disrupted. Thus, the normally greater expression of both Fos and pCREB evoked by viewing novel rather than familiar pictures was disrupted by A-CREB. Hence, the results establish a parallelism of effects across behavioral, systems, and cellular levels of analysis.

A true parallelism across the levels of analysis requires similar temporal profiles for the effects seen at different levels. Here, impairments in recognition memory, differential neuronal activation (measured by Fos and pCREB) by novel and familiar stimuli, and perirhinal synaptic plasticity were found at long delays. In contrast, familiarity discrimination was unimpaired when the delay interval was $15 \mathrm{~min}$. Correspondingly, at $15 \mathrm{~min}$ after induction, expression of LTP was still apparent. Nevertheless, although there was significant LTP 15 min after induction, its level was reduced compared with controls. Some hippocampal studies have also found that interfering with the function of CREB results in an immediate impairment of LTP, although the precise effect depends on the experimental conditions (Bourtchuladze et al., 1994; Matsushita et al., 2001; Pittenger et al., 2002; Balschun et al., 2003; Nguyen and Woo, 2003). The implication for the present experiments is that reductions in short-term perirhinal plasticity, as mirrored in the reduction in LTP amplitude seen at a 15 min delay, is insufficient to produce an impairment in recognition memory, whereas the impairment in perirhinal plasticity mirrored by the loss of LTP by $45 \mathrm{~min}$ may be sufficient to produce memory impairment (impairment of other, unexplored plastic processes cannot be excluded). A possible explanation for the difference at the two time points is that shorter-term recognition memory is supported by an alternative plastic process that is not needed in the production of LTP in vitro and does not involve CREB phosphorylation. Although the primary change underlying perirhinal familiarity discrimination has been sug- 
gested by computational modeling to be synaptic weakening such as occurs in LTD ( Brown and Bashir, 2002; Bogacz and Brown, 2003), the modeling indicates that increases in synaptic strength such as that which occurs with LTP are also necessary for the network to operate efficiently. LTD- and LTP-like mechanisms are both needed if the network is to maintain its excitability across time: synaptic enhancements are essential to counterbalance reductions in synaptic efficacy (Bogacz and Brown, 2003). Accordingly, a facilitatory mechanism, such as is involved in LTP, is also essential for network operation and, hence, plausibly, its disruption will impair familiarity discrimination.

The highly significant reduction $(\sim 40 \%)$ in overall pCREB levels in the A-CREB-expressing animals demonstrates the effectiveness of the adenoviral A-CREB treatment. This reduction was attributable to expression of A-CREB because the comparison was with adenoviral treatment in which only EGFP was expressed. The reduction may be explained because A-CREB does not contain a phosphorylation site that could bind the pCREB antibody used in the immunohistochemistry. Accordingly, one antibody molecule will be bound by the dimer containing A-CREB rather than two by a dimer of native CREB. The total amount of pCREB staining will therefore be lower, and this may be expected to result in a reduction in counts as the staining of some nuclei will then fall below the counting threshold. The reduction did not compromise the finding of a disruption of the normal differential expression produced by novel and familiar stimuli, because such disruption was found for both normalized and absolute counts. The selectivity of the effect to pCREB is emphasized by the absence of a significant overall effect on Fos counts (reduced by only $4 \%$ ) when no account was taken of the effects of stimulus repetition. The lack of effect on Fos provides evidence for the continuing viability of the neurons in the transduced region. Nevertheless, a reduction might have been expected given previous reports that CREB phosphorylation leads to c-fos activation (Ahn et al., 1998; Silva et al., 1998). However, Ahn et al. (1998) found that c-fos induction by $\mathrm{Ca}^{2+}$ and nerve growth factor was only partially blocked by A-CREB (although induction by glutamate was fully blocked). Moreover, NMDA and kainate receptor activation has been reported to lead to increases in Fos without accompanying CREB phosphorylation (Yoneda et al., 1999). These results and our own findings lead to the suggestion that an alternative pathway that does not involve CREB phosphorylation supports the essentially unchanged basal levels of Fos production but not the sensory stimulus-evoked Fos production. Our results indicate that the CREB phosphorylation pathway remains necessary for differential visually evoked Fos differences. The reduction in pCREB counts was similar in both $\mathrm{PRH}$ and Te2. Moreover, A-CREB produced a similar disruption of the normally greater expression of Fos and PCREB produced by novel than familiar stimuli in both $\mathrm{Te} 2$ and $\mathrm{PRH}$. This parallelism of effects in the two areas is consistent with the histological evidence that the adenoviral injections involved both $\mathrm{PRH}$ and Te2. The parallelism of the effects of A-CREB on differential pCREB and Fos expression produced by novel and familiar stimuli is consistent with the known links between CREB phosphorylation and c-fos activation (Ahn et al., 1998; Silva et al., 1998).

That the impairments observed were produced by means other than those arising from interference with CREB phosphorylation may be excluded. Thus, the transduction was localized to perirhinal cortex and Te2. No gross behavioral impairments (beyond that in recognition memory) were observed. In addition, histological examination revealed no gross disruption of the perirhinal cortical tissue nor consistent involvement of any other region, including the hippocampus. The comparisons were made between animals that had been injected with A-CREB and control adenoviral vectors, so that the transduction itself may be excluded from being responsible for the behavioral, Fos, pCREB, and plasticity impairments. Previous studies have also shown that adenoviral vectors elicit a negligible immune response when measured several weeks after injection at the titers used in the present study (Byrnes et al., 1995; Geddes et al., 1996; Gerdes et al., 2000). The behavioral effects clearly originate in the perirhinal cortex and/or $\mathrm{Te} 2$, because that is the region that was virally transduced and the region in which differential Fos and pCREB expression was disrupted. Moreover, A-CREB-expressing slices of perirhinal cortex displayed abnormal LTP. Although disruption of perirhinal cortical plasticity may be sufficient to explain the impairment in recognition memory, the results leave open the possibility that transduction of $\mathrm{Te} 2$ also contributes to the amnesia.

In summary, adenoviral transduction of the perirhinal cortex (and neighboring Te2) with A-CREB impaired the preferential exploration of novel over familiar objects at a long $(24 \mathrm{~h})$ but not a short (15 min) delay, disrupted the normal reduced activation of perirhinal neurons to familiar compared with novel pictures as measured by Fos and pCREB expression, and impaired LTP in perirhinal slices. The consistency of these effects across the behavioral, systems, and cellular levels of analysis provides strong evidence for importance of CREB phosphorylation to synaptic plastic processes within the perirhinal cortex necessary for longterm recognition memory.

\section{References}

Ahn S, Olive M, Aggarwal S, Krylov D, Ginty DD, Vinson C (1998) A dominant-negative inhibitor of CREB reveals that it is a general mediator of stimulus-dependent transcription of c-fos. Mol Cell Biol 18:967-977.

Ahn S, Ginty DD, Linden DJ (1999) A late phase of cerebellar long term depression requires activation of CAMKIV and CREB. Neuron 23:559-568.

Anderson WW, Collingridge GL (2001) The LTP program: a data acquisition program for on-line analysis of long-term potentiation and other synaptic events. J Neurosci Methods 108:71-83.

Balschun D, Wolfer DP, Gass P, Mantamadiotis T, Welzl H, Schutz G, Frey JU, Lipp HP (2003) Does cAMP response element-binding protein have a pivotal role in hippocampal synaptic plasticity and hippocampusdependent memory? J Neurosci 23:6304-6314.

Bogacz R, Brown MW (2003) Comparison of computational models of familiarity discrimination in the perirhinal cortex. Hippocampus 13:494-524.

Bogacz R, Brown MW, Giraud-Carrier C (2001) Model of familiarity discrimination in the perirhinal cortex. J Comput Neurosci 10:5-23.

Bourtchuladze R, Frenguelli B, Blendy J, Cioffi D, Schutz G, Silva AJ (1994) Deficient long-term memory in mice with a targeted mutation of the cAMP-responsive element-binding protein. Cell 79:59-68.

Bozon B, Kelly A, Josselyn SA, Silva AJ, Davis S, Laroche S (2003) MAPK, CREB and zif268 are all required for the consolidation of recognition memory. Philos Trans R Soc Lond B Biol Sci 358:805-814.

Brown MW, Aggleton JP (2001) Recognition memory: what are the roles of the perirhinal cortex and hippocampus? Nat Rev Neurosci 2:51-61.

Brown MW, Bashir ZI (2002) Evidence concerning how neurones of the perirhinal cortex may effect familiarity discrimination. Philos Trans R Soc Lond B Biol Sci 357:1083-1095.

Brown MW, Xiang JZ (1998) Recognition memory: neuronal substrates of the judgement of prior occurrence. Prog Neurobiol 55:149-189.

Brown MW, Wilson FAW, Riches IP (1987) Neuronal evidence that inferomedial temporal cortex is more important than hippocampus in certain processes underlying recognition memory. Brain Res 409:158-162.

Buffalo EA, Ramus SJ, Squire LR, Zola SM (2000) Perception and recognition memory in monkeys following lesions of area TE and perirhinal cortex. Learn Mem 7:375-382. 
Byrnes AP, Rusby JE, Wood MJA, Charlton HM (1995) Adenovirus gene transfer causes inflammation in the brain. Neuroscience 66:1015-1024.

Cho K, Kemp N, Noel J, Aggleton JP, Brown MW, Bashir ZI (2000) A new form of long-term depression in the perirhinal cortex. Nat Neurosci 3:150-156.

Dalley JW, Thomas KL, Howes SR, Tsai TH, Aparicio-Legarza MI, Reynolds GP, Everitt BJ, Robbins TW (1999) Effects of excitotoxic lesions of the rat prefrontal cortex on CREB regulation and presynaptic markers of dopamine and amino acid function in the nucleus accumbens. Eur J Neurosci 11:1265-1274.

Deisseroth K, Bito H, Tsien RW (1996) Signaling from synapse to nucleus: postsynaptic CREB phosphorylation during multiple forms of hippocampal synaptic plasticity. Neuron 16:89-101.

Dix SL, Aggleton JP (1999) Extending the spontaneous preference test of recognition: evidence of object-location and object-context recognition. Behav Brain Res 99:191-200.

Ennaceur A, Aggleton JP (1997) The effects of neurotoxic lesions of the perirhinal cortex combined to fornix transection on object recognition memory in the rat. Behav Brain Res 88:181-193.

Gaffan D, Murray EA (1992) Monkeys (Macaca fascicularis) with rhinal cortex ablations succeed in object discrimination learning despite 24-hr intertrial intervals and fail at matching to sample despite double sample presentations. Behav Neurosci 106:30-38.

Geddes BJ, Harding TC, Hughes DS, Byrnes AP, Lightman SL, Conde G, Uney JB (1996) Persistent transgene expression in the hypothalamus following stereotaxic delivery of a recombinant adenovirus: suppression of the immune response with cyclosporin. Endocrinology 137:5166-5169.

Geddes BJ, Harding TC, Lightman SL, Uney JB (1997) Long-term gene therapy in the CNS: reversal of hypothalamic diabetes insipidus in the Brattleboro rat by using an adenovirus expressing arginine vasopressin. Nat Med 3:1402-1404.

Genoux D, Haditsch U, Knobloch M, Michalon A, Storm D, Mansuy IM (2002) Protein phosphatase 1 is a molecular constraint on learning and memory. Nature 418:970-975.

Gerdes CA, Castro MG, Lowenstein PR (2000) Strong promoters are the key to highly efficient, noninflammatory and noncytotoxic adenoviralmediated transgene delivery into the brain in vivo. Mol Ther 2:330-338.

Glover CPJ, Heywood DJ, Bienemann AS, Deuschle U, Kew JN, Uney JB (2004) Adenoviral expression of CREB protects neurons from apoptotic and excitotoxic stress. NeuroReport 15:1171-1175.

Guzowski JF, McGaugh JL (1997) Antisense oligodeoxynucleotidemediated disruption of hippocampal cAMP response element binding protein levels impairs consolidation of memory for water maze training. Proc Natl Acad Sci USA 94:2693-2698.

Harding TC, Geddes BJ, Murphy D, Knight D, Uney JB (1998) Switching of transgene expression in the brain using an adenoviral tetracyclineregulatable system. Nat Biotechnol 16:553-555.

Holscher C, Rolls ET, Xiang J-Z (2004) Perirhinal cortex neuronal activity related to long-term familiarity memory in the macaque. Eur J Neurosci 18:2037-2046.

Kida S, Josselyn SA, de Ortiz SP, Kogan JH, Masushige S, Silva AJ (2002) CREB required for the stability of new and reactivated fear memories. Nat Neurosci 5:348-355.

Kogan JH, Frankland PW, Silva AJ (2000) Long-term memory underlying hippocampus-dependent social recognition in mice. Hippocampus 10:47-56.

Lamprecht R, Hazvi S, Dudai Y (1997) Lamprecht R, Hazvi S, Dudai Y (1997) cAMP response element binding protein in the amygdala is required for long- but not short-term conditioned taste aversion memory. J Neurosci 17:8443-8450.

Liu F-C, Graybiel AM (1996) Spatiotemporal dynamics of CREB phosphorylation: transient versus sustained phosphorylation in the developing striatum. Neuron 17:1133-1144.

Matsushita M, Tomizawa K, Moriwaki A, Li S-T, Terada H, Matsui H (2001) A high-efficiency protein transduction system demonstrating the role of PKA in long-lasting long-term potentiation. J Neurosci 21:6000-6007.

McCabe BJ, Horn G (1994) Learning-related changes in Fos-like immuno- reactivity in the chick forebrain after imprinting. Proc Natl Acad Sci USA 91:11417-11421.

McMenamin MM, Lantos T, Carter EE, Hamilton L, Charlton HM, Gonzalez SC, Wood MJA (2004) Neuropathological consequences of delivering an adenoviral vector in the rat brain. J Gene Med 6:740-750.

Meunier M, Bachevalier J, Mishkin M, Murray EA (1993) Effects on visual recognition of combined and separate ablations of the entorhinal and perirhinal cortex in rhesus monkeys. J Neurosci 13:5418-5432.

Meunier M, Hadfield W, Bachevalier J, Murray EA (1996) Effects of rhinal cortex lesions combined with hippocampectomy on visual recognition memory in rhesus monkeys. J Neurophysiol 75:1190-1205.

Miller EK, Desimone R (1994) Parallel neuronal mechanisms for shortterm memory. Science 263:520-522.

Mumby DG, Pinel JPJ (1994) Rhinal cortex lesions and object recognition in rats. Behav Neurosci 108:11-18.

Murray EA (1996) What have ablation studies told us about the neural substrates of stimulus memory? Semin Neurosci 8:13-22.

Neve RL (1993) Adenovirus vectors enter the brain. Trends Neurosci 16:251-253.

Nguyen PV, Woo NH (2003) Regulation of hippocampal synaptic plasticity by cyclic AMP-dependent protein kinases. Prog Neurobiol 71:401-437.

Pittenger C, Huang YY, Paletzki RF, Bourtchouladze R, Vronskaya S, Kandel ER (2002) Reversible inhibition of CREB/ATF transcription factors in region $\mathrm{CA} 1$ of the dorsal hippocampus disrupts hippocampus-dependent spatial memory. Neuron 34:447-462.

Silva AJ, Kogan JH, Frankland PW, Kida S (1998) CREB and memory. Annu Rev Neurosci 21:127-148.

Suzuki WA, Zola-Morgan S, Squire LR, Amaral DG (1993) Lesions of the perirhinal and parahippocampal cortices in the monkey produce longlasting memory impairment in the visual and tactual modalities. J Neurosci 13:2430-2451.

Umegaki H, Chernak JM, Ikari H, Roth GS, Ingram DK (1997) Rotational behavior produced by adenovirus-mediated gene transfer of dopamine D-2 receptor into rat striatum. NeuroReport 8:3553-3558.

Wan H, Aggleton JP, Brown MW (1999) Different contributions of the hippocampus and perirhinal cortex to recognition memory. J Neurosci 19:1142-1148.

Wan H, Warburton EC, Zhu XO, Koder TJ, Park Y, Aggleton JP, Cho K, Bashir ZI, Brown MW (2004) Benzodiazepine impairment of perirhinal cortical plasticity and recognition memory. Eur J Neurosci 20:2214-2224.

Warburton E, Koder T, Cho K, Massey PV, Duguid G, Barker GRI, Aggleton JP, Bashir ZI, Brown MW (2003) Cholinergic neurotransmission is essential for perirhinal cortical plasticity and recognition memory. Neuron 38:987-996.

Winters BD, Forwood SE, Cowell RA, Saksida LM, Bussey TJ (2004) Double dissociation between the effects of peri-postrhinal cortex and hippocampal lesions on tests of object recognition and spatial memory: heterogeneity of function within the temporal lobe. J Neurosci 24:5901-5908.

Xiang JZ, Brown MW (1998) Differential neuronal encoding of novelty, familiarity and recency in regions of the anterior temporal lobe. Neuropharmacology 37:657-676.

Yoneda Y, Ogita K, Azuma Y, Ikeda M, Tagami H, Manabe T (1999) $N$-methyl-D-aspartate signaling to nuclear activator protein-1 through mechanisms different from those for kainate signaling in murine brain. Neuroscience 90:519-533.

Zhu XO, Brown MW, McCabe BJ, Aggleton JP (1995) Effects of novelty or familiarity of visual stimuli on the expression of the immediate early gene c-fos in rat brain. Neuroscience 69:821-829.

Zhu XO, McCabe BJ, Aggleton JP, Brown MW (1996) Mapping visual recognition memory through expression of the immediate early gene c-fos. NeuroReport 7:1871-1875.

Ziakopoulos Z, Brown MW, Bashir ZI (1999) Input- and layer-dependent synaptic plasticity in the rat perirhinal cortex in vitro. Neuroscience 92:459-472.

Zola-Morgan S, Squire LR, Amaral DG, Suzuki WA (1989) Lesions of perirhinal and parahippocampal cortex that spare the amygdala and hippocampal formation produce severe memory impairment. J Neurosci 9:4355-4370. 